\title{
La Unión Europea frente a las emergencias de salud pública mundial. La crisis de la COVID-19
}

\section{The European Union in the Face of Global Public Health Emergencies. The COVID-19 Crisis}

\author{
María Teresa Ponte Iglesias ${ }^{1}$ \\ Universidad de Santiago de Compostela (España)
}

Recibido: 15-09-20

Aceptado: 11-10-20

\section{Resumen}

El presente estudio intenta valorar si la Unión Europea ha actuado rápida y adecuadamente en la crisis de la COVID-19. Con este objetivo, partiendo del examen de las bases jurídicas y los mecanismos de coordinación que han conducido a que la salud pública se convirtiera en una de las grandes prioridades de la Unión a escala europea e internacional, pretende aportar elementos de información sobre algunas de las medidas adoptadas hasta ahora por las principales Instituciones europeas con el fin de contener la expansión del virus. Finalmente, se brinda un reflexión final sobre como la actual pandemia exige a la UE estar preparada para futuras crisis sanitarias.

Palabras-clave: COVID-19, OMS, pandemia, salud, UE.

\begin{abstract}
This study attempts to assess whether the European Union has acted quickly and appropriately in the COVID-19 crisis. With this objective, starting from the examination of the legal bases and the coordination mechanisms that have led to public health becoming one of the Union's great priorities at European and international level, it aims to provide information on some of the measures adopted so far by the main European institutions in order to contain

\footnotetext{
${ }^{1}$ mteresa.ponte@usc.es). Catedrática de Derecho Internacional Público y Relaciones Internacionales. Coordinadora del Grupo de Estudios Internacionales (GRESIN) de la USC.

ORCID: https://orcid.org/0000-0002-7771-7005.
} 
the spread of the virus. Finally, a final reflection is provided on how the current pandemic requires the EU to be prepared for future health crises.

Key-words: COVID-19, WHO, Pandemic, Health, EU.

\title{
1. Introducción
}

Con ocasión de la Cumbre Económica Mundial celebrada en febrero de 2018 en los Emiratos Árabes Unidos, el Director General de la OMS, Dr. Tedros Adhanom Ghebreyesus, comenzaba su intervención ilustrándola con una historia en la que parecía relatar una pesadilla del futuro, pero fue exactamente lo que ocurrió durante la epidemia de gripe "española" en 1918, que mató a más gente que la Primera Guerra Mundial en sí misma. Como muy bien señalaba:

\begin{abstract}
(...) sus enseñanzas son tan pertinentes hoy como entonces: una epidemia devastadora puede comenzar en cualquier momento en cualquier país y matar a millones de personas porque no estamos preparados. Porque seguimos siendo vulnerables.

Afortunadamente, desde entonces no hemos vivido una emergencia de salud pública de esa magnitud. Pero puede ocurrir en cualquier momento. Las epidemias son una realidad y el mundo sigue siendo vulnerable.

$[\ldots]$

No sabemos cuando tendrá lugar la próxima pandemia, pero sí sabemos que se cobrará un precio terrible, tanto en vidas humanas como en la economía mundial. Hasta puede que sea causa de inestabilidad política ${ }^{2}$.
\end{abstract}

A continuación, el Director General planteaba a los asistentes a la Cumbre la siguiente cuestión: "¿podemos conseguir un mundo sin pandemias? No hay ninguna garantía, pero con una preparación meticulosa y una respuesta rápida podemos evitar que se descontrolen la mayoría de los brotes y reducir el impacto de aquellos que tengan una propagación internacional"3.

\footnotetext{
${ }^{2}$ ¿Podemos conseguir un mundo sin pandemias?, pp. 1-2, disponible en https://www.who.int/dg/ speeches/2018/pandemic-free-world/es/

${ }^{3}$ Ibid., p. 5. En otro discurso, pronunciado el 8 de julio de 2017, el Director General se expresaba en similares términos: "No sabemos dónde ocurrirá la próxima pandemia mundial, ni cuándo, pero sabemos que tendrá un alto costo humano y económico. Con los viajes aéreos (3000 millones de viajeros anualmente) la propagación mundial de cualquier nuevo patógeno sería cuestión de horas. Además del indecible sufrimiento humano, las pérdidas económicas se medirían en billones: pérdidas para el turismo, el comercio, la confianza de los consumidores, además de los problemas y retos políticos. Habrá dos epidemias: una provocada por el virus y otra por el miedo", Las emergencias sanitarias constituyen uno de los mayores riesgos para la economía y seguridad mundiales, disponible en https://www.who.int/es/dg/speeches/detail/health-emergencies-represent-some-of-the-greatestrisks-to-the-global-economy-and-security
} 
Veintidós meses después la anunciada epidemia se iniciaba en la ciudad china de Wuhan ${ }^{4}$ extendiéndose rápidamente al resto del mundo ${ }^{5}$. Es cierto que en las últimas décadas ha habido otras epidemias -algunas de ellas causadas por otros coronavirus ${ }^{6}$-, cuya aparición constituyó una amenaza grave; sin embargo, estas epidemias -en palabras de Martínez Hernández, Especialista en Medicina Preventiva y Salud Pública-, no conmocionaron de un modo intenso a los ciudadanos y las autoridades de todos los países, todas se extendieron rápidamente por medio del transporte aéreo y todas ocasionaron menoscabo económico y descrédito de las autoridades, con merma de la confianza de las sociedades en sus instituciones y en la administración. Asimismo, todas tuvieron un fuerte impacto mediático, siendo portada de medios de comunicación durante semanas y desencadenando ceses de responsables políticos", pero lo cierto es que "ninguna de ellas ha comprometido la existencia humana en su conjunto ni ha tenido una elevada mortalidad (número total de fallecidos dividido por la población) en términos cuantitativos"7.

Nos encontramos, por tanto, ante un nuevo coronavirus que se ha propagado vertiginosamente por todas las regiones del planeta con una alta tasa de letalidad

${ }^{4}$ El 16 de diciembre el hospital "Wuhan Union" detecta el caso de una mujer china de 57 años que presentaba síntomas de una neumonía atípica de origen desconocido. En los días siguientes, aparecieron otras personas con síntomas similares. El 31 de diciembre la Comisión Municipal de Salud y Sanidad de Wuhan informaba sobre 27 casos, en su mayoría trabajadores del mercado local de Huanen, con inicios de síntomas el 8 de diciembre. Una semana después, el 7 de enero de 2020, Pekín comunicaba a la OMS que había logrado aislar e identificar el genoma del virus, poniendo a disposición de la comunidad internacional esta información para el desarrollo de test de prevención y la búsqueda de una vacuna, disponible en http://www.chinacdc.cn/en

${ }^{5}$ Aunque en los primeros días los brotes de la enfermedad se limitaron al continente asiático, pronto el virus se extendió a Europa, Oriente Medio y el continente americano, con un total de 39.801.612 casos confirmados en el mundo a fecha del 20 de octubre - 7.447.952 se localizan en Europa-y un saldo de más de 1.000.000 de fallecidos a nivel global. Cifras globales y casos COVID-19 por país, en https://covid19. who.int Una cronología y extensión de la enfermedad ver Alberto Priego, COVID-19, una radiografia de la pandemia, Documento de opinión IEEE 06/2020, disponible en http://www. ieee.es/Galerias/fichero/docs marco/2020/DIEEEM06 2020ALBPRI COVIDRadio.pdf

${ }^{6}$ Entre ellos, el SARS-CoV, identificado, por primera vez, a finales de febrero de 2003, tras el brote del Síndrome Respiratorio Agudo y Severo (SARS), que comenzó en el año 2002 en Asia e infectó a más de 8.000 personas. En septiembre de 2012, se identificó en Arabia Saudí un nuevo tipo de coronavirus denominado Síndrome Respiratorio de Oriente Medio (MERS). Su aparición dio lugar a una nueva alerta sanitaria mundial por parte de la OMS. Sobre el particular ver David Alejandro, Cabrera-Gaytán, Alfredo Vargas-Valerio, Concepción Grajales-Muñiz, "Infección del nuevo coronavirus: nuevos retos, nuevos legados", en Revista Médica del Instituto Mexicano del Seguro Social, 2014, 452(4), pp. 438-41. Coronavirus: COVID-19. Informe Técnico, Consejo General del Colegio de Farmacéuticos, marzo, 2020.

Otros brotes importantes han sido el Ebola en 2014 o el virus Zika en 2015. Ver al respecto José Luis de la Flor, "La seguridad sanitaria global a debate. Lecciones críticas aprendidas de la 24. ${ }^{\circ}$ EVE", en Cuestiones de seguridad internacional en el África del siglo XXI Comillas Journal of International Relations, Núm. 13 (2018), pp. 49-62; OMS, Enfermedad por el virus del Ebola, 10 de febrero de 2020, disponible en https://www.who.int/es/news-room/fact-sheets/detail/ebola-virus-disease

7 Juan Martínez Hernández, "Pandemias y bioamenazas globales del siglo XXI", ARI no 42/2016, Real Instituto Elcano, 30/VI/2016, p. 2, disponible en http://www.realinstitutoelcano.org/wps/portal/ rielcano es/contenido?WCM_GLOBAL_CONTEXT=/elcano/elcano_es/zonas_es/ari42-2016martinezhernandez-pandemias-bioamenazas-globales-siglo-21

Araucaria. Revista Iberoamericana de Filosofí, Política, Humanidades y Relaciones Internacionales, año $22, \mathrm{n}^{\circ} 45$. Tercer cuatrimestre de 2020. Pp. 337-357. ISSN 1575-6823 e-ISSN 2340-2199 https://dx.doi.org/10.12795/araucaria.2020.i45.14 
entre los mayores y para el que de momento más allá de las medidas de prevención (higiene de manos, distanciamiento, uso de mascarillas, cuarentena, aislamiento) no se conoce tratamiento efectivo. Su propagación nos sitúa frente a una de las mayores emergencias de salud pública, que ha puesto y continúa poniendo a prueba los sistemas sanitarios de todo el mundo, la capacidad de los gobiernos en la preparación y respuesta frente a la pandemia, las economías y al conjunto de la población con un fuerte impacto sobre su vida y sus libertades individuales ${ }^{8}$. Como ha subrayado el Secretario General de la ONU nos enfrentamos con un "virus que no discrimina, pero sus efectos si; saca a la luz las profundas deficiencias en la prestación de servicios públicos y las desigualdades estructurales que obstaculizan el acceso a ellos". Esta pandemia es mucho más que una emergencia de salud pública, "es una crisis económica. Una crisis social. Y una crisis humana que se está convirtiendo rápidamente en una crisis de derechos humanos"9.

Este nuevo coronavirus bautizado como COVID-19 (2019-nCoV) determinó que el Director General de la OMS, a recomendación del Comité de Emergencias del Reglamento Sanitario Internacional (2005), tomase la decisión final de comunicar que el brote cumplía los criterios para declarar una emergencia de salud pública de importancia internacional ${ }^{10}$. Y dos meses después, la OMS llegaba a la conclusión de que la COVID-19 se consideraba una pandemia ${ }^{11}$.

${ }^{8}$ Sobre el particular ver Juan Manuel Faramiñan Gilbert, "La protección de la salud pública y el respeto de las libertades individuales ante el COVID-19", Freedom, Security \& Justice: European Legal Studies. Rivista quadrimestrale on line sullo Spazio europeo di libertà, sicurezza e giustizia, 2020, n. 2, pp. 1-21, disponible en http://www.fsjeurostudies.eu

9 Todos estamos juntos en esto: los derechos humanos y la respuesta y recuperación ante la COVID-19, 04/23/2020, disponible en https://www.un.org/sg/es/content/sg/statement/2020-04-23/ we-are-all-together-human-rights-and-covid-19-response-and-recovery-video-message-thesecretary-general-delivered

${ }^{10}$ Declaración sobre la segunda reunión del Comité de Emergencias del Reglamento Sanitario Internacional (2005) acerca del brote del nuevo coronavirus (2019-nCoV), 30 de enero de 2020. En esta Declaración, el Comité advierte a los Estados que "todos deben estar preparados para adoptar medidas de contención, como la vigilancia activa, la detección temprana, el aislamiento y el manejo de los casos, el seguimiento de contactos y la prevención de la propagación del 2019-nCoV, y para proporcionar a la OMS todos los datos pertinentes". Asimismo, "deben procurar, principalmente, reducir la infección en las personas, evitar la transmisión secundaria y la propagación internacional y colaborar con la respuesta internacional mediante la comunicación y la colaboración multisectoriales y la participación activa para incrementar los conocimientos sobre el virus y la enfermedad, y para impulsar las investigaciones." Además, les recuerda que "están jurídicamente obligados a compartir información con la OMS en virtud del RSI.", disponible en https:/www.who.int/es/news-room/ detail/30-01-2020-statement-on-the-second-meeting-of-the-international-health-regulations-(2005)emergency-committee-regarding-the-outbreak-of-novel-coronavirus-(2019-ncov)

${ }^{11}$ Alocución de apertura del Director General de la OMS en la rueda de prensa sobre la COVID-19 celebrada el 11 de marzo de 2020, disponible en https://www.who.int/es/dg/speeches/detail/who-directorgeneral-s-opening-remarks-at-the-media-briefing-on-covid-19-11-march-2020. La declaración de pandemia se basó en dos razones fundamentales: la velocidad y la escala de la transmisión (notificación a la OMS de casi 125.000 contagios en 118 países y territorios) y en el hecho de que algunos países no están abordando esta amenaza con el nivel de compromiso político necesario para controlarla.

A juicio del Director General "Todos los países deben encontrar un delicado equilibrio entre la protección de la salud, la prevención de los trastornos sociales y económicos, y el respeto de los derechos 
Un panorama éste, a tenor del cual se impone hoy más que nunca la solidaridad y cooperación de la Comunidad internacional "para prestarse mutuamente apoyo a fin de determinar la fuente de este nuevo virus, conocer el alcance que puede tener la transmisión interpersonal, estar preparados ante la posible aparición de casos importados y llevar a cabo investigaciones para encontrar el tratamiento necesario" 12 . Así pues, en este contexto es muy importante la acción institucional realizada por la OMS, o en su caso, por organizaciones regionales como la Organización Panamericana de la Salud (OPS) o la propia Unión Europea (UE), en la que vamos a centrarnos a continuación.

\section{Papel de la Unión Europea en la prevención y control de pandemias}

Para valorar si la UE ha actuado rápida y adecuadamente en la crisis de la COVID-19, es necesario atender con carácter previo a dos aspectos fundamentales. Por un lado, los esfuerzos realizados por esta organización internacional de integración para que la salud pública se convirtiera en una de sus grandes prioridades. Y, por el otro, la cooperación de la UE con la OMS y su participación en foros internacionales como la Iniciativa Global para la Seguridad Sanitaria.

\section{A) Bases jurídicas y mecanismos de coordinación}

Atendiendo al primero de estos aspectos advertimos que los temas de salud pública se introdujeron muy tardíamente en los instrumentos constitutivos. Inicialmente los tratados creadores de las Comunidades Europeas carecían de referencias explicitas a una política de protección de la salud pública, si bien podía deducirse de forma indirecta una cierta fundamentación de la acción comunitaria en el antiguo art. 2 del TCE. La progresiva imbricación de la dimensión sanitaria en otros ámbitos comunitarios como las políticas social, agrícola o de medio ambiente a lo largo de los años ochenta y noventa junto con la jurisprudencia del Tribunal de Justicia, facilitaron la aprobación de normas que indirectamente daban cumplimiento a objetivos de protección de salud pública ${ }^{13}$.

Será el Tratado de la Unión Europea de 1992 quien de un paso importante al incorporar un Título X con un único artículo (art.129 del TCE), que introduce la política de protección de la salud pública, instando a la Comunidad Europea

humanos. A tal efecto, propuso una estrategia basada en cuatro pilares: i) prepararse y estar a punto; ii) detectar, prevenir y tratar; iii) reducir y suprimir; iv) innovar y mejorar, en Alocución de apertura del Director General de la OMS en la rueda de prensa para las misiones diplomáticas sobre la COVID-19 celebrada el 12 de marzo de 2020, disponible en https://www.who.int/es/dg/speeches/detail/who-directorgeneral-s-opening-remarks-at-the-mission-briefing-on-covid-19-12-march-2020

${ }^{12}$ Declaración sobre la segunda reunión del Comité de Emergencias del Reglamento..., cit.

${ }^{13}$ Mar Campins Eritja, "La Unión Europea y la protección de la salud pública", en Xavier Pons Rafolds, ed.: Salud Pública y Derecho Internacional, Madrid, Marcial Pons, 2010, p. 378. 
a contribuir "a la consecución de un alto nivel de salud humana fomentando la cooperación entre los Estados miembros y, si fuere necesario, apoyando la acción de los mismos" en una serie de áreas específicas entre las que destaca "la prevención de las enfermedades, especialmente las más graves y ampliamente difundidas", apoyando la investigación de su etiología y de su transmisión, así como la información y la educación sanitarias. De esta manera, queda delimitado el marco de acción y las funciones de los Estados miembros y de la Unión, presentando la política de salud pública un carácter complementario y subsidiario frente a la actuación de los Estados miembros ${ }^{14}$.

Con el Tratado de Lisboa se refuerza la importancia de la salud pública en la Unión Europea y el control de los brotes epidémicos y de las situaciones de crisis adquieren una mayor significación. El nuevo art. 168 del TFUE incluye entre las acciones de la UE "la vigilancia de las amenazas transfronterizas graves para la salud, la alerta en caso de tales amenazas y la lucha contra ellas", el fomento de "la cooperación entre los Estados miembros destinada a mejorar la complementariedad de sus servicios de salud en las regiones fronterizas" y la coordinación de "iniciativas tendentes a establecer orientaciones e indicadores, organizar el intercambio de mejores prácticas y preparar los elementos necesarios para el control y la evaluación periódicos". Además, se amplia la competencia de la UE para adoptar tanto medidas de fomento encaminadas a "proteger y mejorar la salud humana y, en particular, a luchar contra las pandemias transfronterizas, como "medidas relativas a la vigilancia de las amenazas transfronterizas graves para la salud, a la alerta en caso de tales amenazas y a la lucha contra las mismas"15.

14 "Aunque el texto del artículo 129 no menciona expresamente este principio, la política de salud pública, lejos de constituir una política de competencia exclusiva de la Comunidad y como competencia compartida con los Estados miembros, se encuentra íntimamente unida al principio de subsidiariedad, ello, por lo demás, se extrae con claridad del pár. 11 del art. 129.1 del TCE, cuándo dice que la Comunidad fomentará la cooperación de los Estados miembros y solamente si fuere necesario apoyará su acción. Además, conforme al art. 129.4, si bien el Consejo puede adoptar acciones, éstas se caracterizarán por "contribuir a la consecución de los objetivos del presente artículo" y favorecer el esfuerzo de los Estados miembros. En este marco, la Comisión facilita la coordinación de los programas de los Estados (arts. 129.1 y 2), y el Consejo sólo puede, a propuesta de la Comisión, adoptar recomendaciones por mayoría cualificada o bien, con arreglo al procedimiento previsto en el art. 189B, es decir, al procedimiento de codecisión, y previa consulta al Comité Económico y Social y al Comité de las Regiones, adoptar medidas de fomento, con exclusión de toda armonización de las disposiciones legales y reglamentarias de los Estados miembros", en José Manuel Sobrino Heredia, "La política de salud pública en la Unión Europea", Anuario da Facultade de Dereito da Universidade da Coruña, 1998, p. 553.

El nuevo Tratado de Ámsterdam modificara en parte el artículo 129 TCE, para establecer que, en la definición de cualquier política y acción comunitaria, la UE debe "garantizar" un alto nivel de protección de la salud humana".

${ }^{15}$ Con todo, no hay que dejar de recordar que "poco se ha avanzado sin embargo en lo que se refiere al desarrollo o al reforzamiento de la competencia de la Unión Europea es esta materia..., el nuevo Tratado confirma el carácter meramente complementario de la acción de la Unión Europea en los arts. 4 y 6 TFUE. Además, el art. 168.7 TFUE sigue reservando a los Estados miembros la "definición de su política de salud, así como la organización y prestación de servicios sanitarios y atención médica", que ahora incluye expresamente también "la gestión de los servicios de salud y de atención médica, así como la asignación de los recursos que se destinan a dichos servicios”, en Campins Eritja: op.cit., p. 384. 
Pero lo cierto es que la salud continua siendo competencia exclusiva de los Estados miembros y por tanto la capacidad de la UE para hacer frente a la expansión de virus, como el COVID-19, se limita de conformidad con el Tratado de Lisboa a una "combinación de mecanismos de vigilancia y alerta temprana, la planificación de respuestas conjuntas ante las posibles epidemias, la recopilación de información y evidencias científicas y la creación de estructuras que permitan compartir esta información con objeto de adoptar las medidas adecuadas de contención de las amenazas epidemiológicas" ${ }^{16}$.

Bajo este modelo la UE se fue preparando para hacer frente a situaciones de amenazas de epidemias y pandemias ${ }^{17}$. En este sentido, trasciende la Decisión $\mathrm{N}^{\mathrm{o}} 1082 / 2013 / \mathrm{UE}$ sobre las amenazas transfronterizas graves para la salud dirigida a mejorar la preparación y reforzar la capacidad de respuesta coordinada a las emergencias para la salud en la UE ante posibles pandemias y amenazas transfronterizas futuras, a través de una serie de medidas tales como: el refuerzo de la capacidad de planificación en materia de preparación a nivel de la UE, reforzando la coordinación así como la puesta en común de la información sobre los planes nacionales de preparación; la mejora de la evaluación de riesgos y la gestión de amenazas transfronterizas para la salud; el establecimiento de los mecanismos necesarios para la adquisición conjunta de productos médicos de respuesta sanitaria; una mayor sincronización de la respuesta a escala de la UE mediante un mandato legal sólido para que el Comité de Seguridad Sanitaria coordine la preparación; o una mayor coordinación de la comunicación de riesgos y crisis y el fomento de la cooperación internacional ${ }^{18}$.

Asimismo, para responder a las posibles pandemias y a las amenazas sanitarias transfronterizas, la UE, de manera progresiva, fue creando una serie de mecanismos coordinados de evaluación de riesgos, cuyo asesoramiento

${ }^{16}$ Ibid., p. 385.

${ }_{17}$ Ya en El Libro Blanco "Juntos por la salud: un planteamiento estratégico para la UE (20082013)", presentado por la Comisión Europea el 23 de octubre de 2007, la Comisión manifiesta que existen "ámbitos en los que la sola acción de los Estados miembros no sería eficaz, haciéndose pues indispensable una cooperación a nivel comunitario. Es el caso de las grandes amenazas para la salud y de ciertas cuestiones que tienen un impacto transfronterizo o internacional, como las pandemias". Las pandemias suponen acuciantes amenazas potenciales para la salud... La coordinación y la respuesta rápida a las amenazas para la salud a escala mundial, así como el refuerzo de las capacidades de la CE y de terceros países en este ámbito, son un componente esencial de la misión de la Comunidad en materia de sanidad. Esta dimensión se relaciona con el objetivo estratégico global de seguridad de la Comisión... La lucha contra las pandemias requiere una cooperación a nivel comunitario y una coordinación entre Estados miembros y actores internacionales.", COM (2007) 630 final, Bruselas, 23.10.2007, pp. 2, 3 y 9. Un análisis en Antonio Calvete Oliva, "Estrategia de Salud de la Unión Europea: Salud pública para las personas Europas", Revista Española de Salud Pública, № 3, mayojunio 2008, pp. 273-281. Ver también la Decisión No 1350/2007/CE del Parlamento Europeo y del Consejo de 23 de octubre de 2007 por la que se establece el Segundo Programa de acción comunitaria en el ámbito de la salud (2008-2013), DO, L 301/3, 20.11.2007, párr. 5.

${ }_{18}$ Decisión No 1082/2013/UE del Parlamento Europeo y del Consejo de 22 de octubre de 2013 sobre las amenazas transfronterizas graves para la salud y por la que se deroga la Decisión No 2119/98/ CE, DO, L 293/1, 5.11.2013. 
científico orienta la coordinación de la respuesta, tales como la Red de Vigilancia Epidemiológica y de Control de las Enfermedades Transmisibles en la Comunidad, el Sistema de Alerta Precoz y Respuesta (SAPR), el Comité de Seguridad Sanitaria (CSS), el Centro Europeo para la Prevención y el Control de Enfermedades (ECDC) y el Mecanismo de Respuesta Política Integrada a las Crisis (RPIC).

El primero de estos mecanismos, la Red de Vigilancia Epidemiológica y de Control de las Enfermedades Transmisibles en la Comunidad, tiene como objetivos: i) potenciar la cooperación y la coordinación entre los Estados miembros con la asistencia de la Comisión, a fin de permitir la instauración de un sistema de comunicación continua, bien estructurado, fiable y eficaz a nivel comunitario entre las autoridades nacionales de vigilancia de las enfermedades transmisibles y la Comisión Europea; ii) facilitar la identificación rápida y consensuada de los casos de enfermedades transmisibles que se dan en la Comunidad, o que se dan fuera de ella pero que se han importado o es probable que se importen; iii) ofrecer a las autoridades competentes la oportunidad y los medios de consultarse acerca de las medidas preventivas que conviene adoptar, y ayudar a aplicarlas de forma coordinada y a evaluar su eficacia; y iv) proporcionar a las autoridades apoyo para analizar e interpretar los datos disponibles y poner en práctica las medidas necesarias para impedir que estas enfermedades se propaguen.

La gestión y coordinación de las actividades de la Red corresponde a la Comisión. Dentro de este mandato se incluyen la gestión del sistema de alerta precoz y respuesta, con trascendencia política; y la coordinación técnica de los componentes de la vigilancia epidemiológica ${ }^{19}$.

El Sistema de Alerta Precoz y Respuesta creado por la Comisión Europea en 1999, es un mecanismo que permite a los órganos sanitarios competentes de los Estados miembros enviar alertas sobre incidentes que pueden tener efectos en la UE, compartir información y coordinar su respuesta ${ }^{20}$.

Por su parte, el Comité de Seguridad Sanitaria es un grupo de expertos integrado por representantes de los Estados miembros y de la Comisión,

${ }_{19}$ Decisión No 2119/98/CE del Parlamento Europeo y del Consejo de 24 de septiembre de 1998 por la que se crea una red de vigilancia epidemiológica y de control de las enfermedades transmisibles en la Comunidad, DO L 268, 03/10/1998.

${ }_{20}$ 2000/57/CE: Decisión de la Comisión, de 22 de diciembre de 1999, relativa al sistema de alerta precoz y respuesta para la vigilancia y control de las enfermedades transmisibles en aplicación de la Decisión $n^{\circ}$ 2119/98/CE del Parlamento Europeo y del Consejo, DO L 021, 26/01/2000. Su anexo I recoge los hechos o indicios de los hechos que, por sí solos o asociados con otros, constituyan o puedan llegar a constituir amenazas para la salud pública. Su anexo II expone los procedimientos que han de seguirse para el intercambio de información relativa a indicios sobre hechos; en caso de que el hecho pueda convertirse en una amenaza a la salud pública o cuando el hecho constituya efectivamente una amenaza para la salud pública, respectivamente; y para informar a la población y a los profesionales de los sectores afectados. 
establecido informalmente por el Consejo en el año $2001^{21}$ con la misión de intercambiar información y coordinar las medidas de preparación, respuesta y cooperación internacional sobre las amenazas relacionadas con la salud. En 2013, se procedió a asignarle un mandato definido para evitar duplicidades con otras entidades de la Unión responsables en materia de gestión de riesgos ${ }^{22}$.

En 2005 se crea el Centro Europeo para la Prevención y el Control de Enfermedades. El ECDC es un órgano técnico carente de capacidad ejecutiva, que trabaja en estrecha colaboración con la Comisión, el Parlamento Europeo y las instituciones nacionales de salud pública de los Estados miembros. Su misión se centra fundamentalmente en la preparación frente a pandemias y enfermedades infecciosas mediante la recopilación y difusión de información científico-técnica; el suministro e intercambio de información actualizada a y entre las Instituciones y agencias europeas, los Estados miembros y las organizaciones internacionales con competencias en el ámbito de la salud pública; y el desarrollo e implementación de acciones conjuntas ${ }^{23}$.

Además de los mecanismos ya indicados, la UE cuenta con el dispositivo de Repuesta Política Integrada a la Crisis $^{24}$. Se trata de un mecanismo dirigido

${ }^{21}$ El hecho de que la UE constituya un espacio abierto en el que es esencial la existencia de mecanismos adecuados para garantizar la rapidez de la notificación y del intercambio de información en caso de amenazas y atentados, justificó la creación, en octubre de 2001, del CSS, compuesto por representantes de alto nivel de los Ministros de Sanidad, con la finalidad de favorecer la cooperación en la lucha contra el bioterrorismo. El Comité inicialmente estableció un programa de cooperación en materia de preparación y respuesta en caso de atentados con agentes biológicos y químicos, que giraba en torno a cuatro objetivos, destacando su responsabilidad en materia de información sobre amenazas relacionadas con la salud, planes de preparación y respuesta y estrategias de gestión de crisis, en Comunicación de la Comisión al Consejo y al Parlamento Europeo, de 2 de junio de 2003, relativa a la cooperación en la Unión Europea en materia de preparación y respuesta ante atentados con agentes biológicos y químicos (seguridad sanitaria), COM (2003) 320 final, no publicada en el Diario Oficial.

En 2006, se actualizó su mandato para cubrir el período transitorio hasta 2008. A tal efecto, se establece que "Las funciones centrales del CSS tienen dos vertientes: la preparación y la gestión de las amenazas... El CSS facilita una toma de decisiones políticas coordinadas entre los Estados miembros y a nivel de la UE sobre las cuestiones de sanidad pública que entran dentro de su mandato. Ello incluye una contribución al proceso de aplicación del Reglamento Sanitario Internacional por parte de la OMS, tal como se establece en la Comunicación de la Comisión al Parlamento Europeo y al Consejo sobre el Reglamento Sanitario Internacional", en Comunicación de la Comisión al Consejo sobre la prolongación y la ampliación provisional del mandato del Comité de Seguridad Sanitaria con vistas a una futura revisión general de las estructuras que gestionan las amenazas para la salud a nivel de la UE, COM (2006) 699 final, 16.11.2006

22 Decisión No 1082/2013/UE..., cit. Las funciones del Comité de acuerdo con lo establecido en el art. 17 de la mencionada Decisión son las siguientes: a) prestar apoyo para el intercambio de información entre los Estados miembros y la Comisión acerca de la experiencia adquirida en lo que respecta a la aplicación de la presente Decisión; b) coordinar, en cooperación con la Comisión, las actividades de planificación de la preparación y respuesta de los Estados miembros; y c) coordinar, igualmente en cooperación con la Comisión, la comunicación en situaciones de riesgo y de crisis y las respuestas de los Estados miembros a las amenazas transfronterizas graves para la salud.

23 Daniel J. Catalán Matamoros, "Mecanismos de prevención y control de las enfermedades infecciosas en la Unión Europea: El Centro Europeo para la Prevención y el Control de enfermedades", en Xavier Pons Rafols, ed.: Salud Pública..., cit., pp. 415-424.

${ }^{24}$ Aprobado por el Consejo el 25 de junio de 2013. Cinco años después el Consejo adopta la Decisión de ejecución (UE) 2018/1993 del Consejo de 11 de diciembre de 2018 sobre el dispositivo 
a prestar apoyo cuando se produce una crisis o una catástrofe (natural o provocada), tanto dentro como fuera de la Unión y con efectos de gran alcance o importancia política ${ }^{25}$, que permite que la toma de decisiones en el nivel político de la Unión sea rápida y coordinada entre las Instituciones europeas, los Estados miembros afectados y otros actores. Añadir, que el RPIC se basa en el principio de subsidiariedad, y respeta plenamente las competencias de los Estados miembros en situaciones de crisis, sin sustituir a los dispositivos existentes a nivel sectorial.

El brote de la COVID-19 motivó, como veremos más adelante, que el 28 de enero de 2020, la Presidencia croata decidiera activar este dispositivo RPIC en el modo de puesta en común de información ${ }^{26}$, con objeto de facilitar una interpretación común de la situación entre los Estados miembros y las Instituciones. Dos meses después a la vista de la gravedad de la pandemia y de los diferentes sectores afectados (sanitario, consular, protección civil, económico), la Presidencia decidió pasar al modo de activación plena.

\section{B) Coordinación con otras organizaciones y foros internacionales}

Centrándonos en el segundo de los aspectos apuntados al objeto de valorar si la UE ha actuado rápida y adecuadamente en la crisis de la COVID-19, advertimos como el art. 168.3 del TFUE establece la cooperación de la Unión con organizaciones internacionales competentes en materia de salud pública. En este sentido trasciende, en particular la cooperación con la OMS y su participación en foros internacionales como la Iniciativa Global para la Seguridad Sanitaria.

Aunque las relaciones entre la UE y la OMS se remontan a 1959, lo cierto es que será a partir de 1982 cuando la Comunidad Europea disfrute del estatuto de observador participando regularmente en las sesiones de la Asamblea Mundial de la Salud, del Consejo Ejecutivo y del Comité Regional para Europa. Diez años después, tuvo lugar la apertura en Bruselas de la "Oficina de la OMS ante la Unión Europea”, con el objetivo de favorecer los contactos entre ellas y servir como instrumento a la OMS en esta tarea ${ }^{27}$.

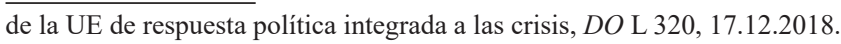

25 El RPIC se ha utilizado para apoyar el intercambio de información sobre crisis complejas (Siria/Irak, Yemen, Ucrania, Nepal, crisis del ébola, etc.), sobre comunicación de crisis, sobre ayuda humanitaria y sobre lucha contra el terrorismo. También se ha utilizado para ejercicios de respuesta de la Unión a crisis graves causadas por ciberataques, catástrofes naturales o amenazas híbridas.

${ }^{26}$ Este dispositivo presenta dos modalidades de activación en función de la gravedad de la crisis y de las necesidades para la respuesta: i) modo de puesta en común de información, que sirve para establecer una imagen clara de la situación y preparar el terreno para una posible activación plena; ii) modo de activación plena, que implica la preparación de medidas de respuesta.

${ }^{27}$ María del Carmen Pérez Bernárdez, Las relaciones de la Unión Europea con organizaciones internacionales: Análisis jurídico de la práctica constitucional, Madrid, Dirección General de 
La intensificación de la cooperación entre ambos organismos se produce tras el Canje de Notas sobre la consolidación e intensificación de la cooperación concluido entre la OMS y la Comisión que pone de relieve el interés común de ambas organizaciones en materia de salud y en los ámbitos relacionados con ésta. Entre los compromisos que adquieren, esta el de responder a las amenazas potenciales para la salud. En materia de cooperación se considera prioritario el tratamiento efectivo de epidemias y amenazas sanitarias y la elaboración de instrumentos y otros documentos de carácter legislativo ${ }^{28}$.

Uno de los ámbitos en lo que se ha reflejado la intensidad de la cooperación entre la Comisión y la OMS es la implementación del Reglamento Sanitario Internacional (RSI 2005). Se trata de un instrumento convencional concebido para ayudar a proteger a todos los Estados contra la propagación internacional de enfermedades ${ }^{29}$ que prevé, entre otros aspectos: i) Derechos y obligaciones para los Estados Partes (y funciones para la OMS) en materia de vigilancia nacional e internacional; ii) Evaluación y respuesta de salud pública; iii) Medidas sanitarias aplicadas por los Estados Partes a los viajeros internacionales, aeronaves, embarcaciones, vehículos automotores y mercancías; y iv) Salud pública en los puertos, aeropuertos y pasos fronterizos terrestres internacionales (designados globalmente como «puntos de entrada»). Uno de los mecanismos esenciales que establece es la obligación de notificación que se extiende a todos los eventos que ocurran en el territorio de un Estado Parte y que puedan constituir una emergencia de salud pública de importancia internacional, así como toda medida sanitaria aplicada en respuesta a esos eventos ${ }^{30}$.

Por su parte, sobre la participación de la UE en la Iniciativa Global para la Seguridad Sanitaria creada en noviembre de 2001, bajo la dirección del antiguo Departamento de Salud y Servicios Sociales de los EE. UU., cumple indicar que se trata de un foro ministerial en el que participan los ministros y secretarios de los países del G7+ y el Comisario de Salud de la Comisión Europea. Solo

\footnotetext{
Universidades, Consejería de Educación, 2003.

${ }^{28}$ DO C 1/7, 4.1.2001. El inciso 1.4 incluido en la parte D del Memorándum del Canje de Notas establece que "el Director General de la Organización Mundial de la Salud podrá, tras consultar a la Comisión de las Comunidades Europeas, llamar la atención del órgano director competente de la Organización Mundial de la Salud sobre la cuestión de la participación de la Comisión en los trabajos de dicho órgano en casos particulares, por ejemplo en la negociación de acuerdos internacionales, y sobre el papel de la Comunidad Europea en el marco de dichos acuerdos.

${ }^{29}$ Se adoptó por la AMS en 1951, siendo enmendado en numerosas ocasiones, la última el 23 de mayo de 2005. Entró en vigor el 15 de junio de 2007, y en la actualidad es jurídicamente vinculante para 196 Estados Partes (incluidos todos los Estados Miembros de la OMS). España es Parte (BOE núm. 62, 12 marzo 2008).

En cuanto a su aplicación en la legislación nacional, incumbe a cada Estado Parte determinar cómo se cumplirán los requisitos del RSI, en función de su sistema jurídico y de gobernanza, su contexto sociopolítico y sus políticas.

${ }^{30}$ Reglamento Sanitario Internacional (2005). Breve introducción a su aplicación en la legislación nacional, enero de 2009, WHO/HSE/IHR/2009.2.
} 
tres Estados de la UE (Alemania, Francia e Italia) son miembros oficiales de la Iniciativa, pero la participación de la Comisión Europea garantiza que los resultados de sus actividades se trasladen a todos los demás Estados miembros a través del Comité de Seguridad Sanitaria. La Organización Mundial de la Salud también está presente, en calidad de asesor técnico.

Aunque esta Iniciativa Global tiene como principal objetivo reducir las potenciales consecuencias que provocaría la diseminación intencionada de un agente biológico de alta transmisibilidad, resulta claro desde el punto de vista que nos ocupa que un brote de un virus de alta transmisibilidad como el COVID-19 en este mundo globalizado constituye sin duda una amenaza a la seguridad global, demostrándose así que los actuales mecanismos existentes para hacer frente al bioterrorismo pueden servir también de modelo en la capacidad de respuesta frente a los riesgos de salud pública de origen natural, ya que los agentes biológicos son los mismos y las medidas de lucha y control son similares ${ }^{31}$.

\section{Actuación de la Unión Europea ante la crisis de la COVID-19}

A la vista de lo hasta aquí expuesto corresponde examinar ahora como ha ido respondiendo la UE a la COVID-19 tras las innumerables críticas recibidas, en las que se le achaca su falta de liderazgo y de escasa capacidad de gestionar las crisis de manera coordinada entre los Estados miembros ${ }^{32}$. A este respecto, convendría tener presente, como ya indicamos, que la salud continúa siendo competencia exclusiva de los Estados miembros, de manera que la capacidad de la UE para hacer frente a la expansión de la pandemia se limita a una combinación de los mecanismos - ya examinados-dirigidos a la adopción de medidas adecuadas para su contención. Por ello, como señala Faramiñán Gilbert, "resulta injusto menospreciar su actividad ya que en el marco de la Comisión Europea se coordina la cooperación y el intercambio de información con los Estados de la Unión y en el caso del coronavirus ha estado interactuando con los gobiernos de los países miembros desde que se

31 Alberto Cique Moya, "Capacidades sanitarias militares en la prevención y lucha frente a emergencias epidémicas”, en Cuadernos de Estrategia 203, Emergencias pandémicas en un mundo globalizado: Amenazas a la seguridad, Madrid, Instituto Español de Estudios Estratégicos, 2020, p. 221, disponible en http://www.ieee.es/publicaciones-new/cuadernos-de-estrategia/2020/ Cuaderno 203.html

${ }^{32}$ En este sentido ver, entre otros, Miguel Ángel Benedicto, Los retos de Europa y su futuro tras la pandemia de la COVID-19. Documento de Opinión IEEE 72/2020, pp. 9 y 13, disponible en http:// www.ieee.es/Galerias/fichero/docs_opinion/2020/DIEEEO72_2020MIGBEN_retosEU.pdf; Ignacio Fuente Cobo, El mundo después de la pandemia: el nuevo orden no será chino. Documento de Opinión IEEE 33/2020, p. 7, disponible en http://www.ieee.es/Galerias/fichero/docs_opinion/2020/ DIEEEO33 2020IGNFUE mundo.pdf;

Andrea Betti, Coronavirus: última llamada para la Unión Europea, disponible en https:// repositorio.comillas.edu/xmlui/handle/11531/45486

Araucaria. Revista Iberoamericana de Filosofia, Politica, Humanidades y Relaciones Internacionales, año $22, \mathrm{n}^{\circ} 45$. Tercer cuatrimestre de 2020. Pp. 337-357. ISSN 1575-6823 e-ISSN 2340-2199 https://dx.doi.org/10.12795/araucaria.2020.i45.14 
lanzó la primera alerta en 9 de enero de 2020. Otra cosa muy diferente es la actitud de cada Estado miembro que, en función de sus propias competencias no transferidas a la Unión en materia de salud pública, haya tenido mayor o menor sensibilidad a la hora de atajar esta pandemia"33.

En esta línea, procede indicar que la Unión Europea ha venido colaborando con los Estados miembros para contener la propagación del virus con el fin de proteger la salud y el bienestar de sus ciudadanos y emplear todos los instrumentos a su alcance. En este sentido, vamos a referirnos brevemente a algunas de las principales actuaciones emprendidas por el Consejo de la Unión Europea y la Comisión.

Comenzando por el Consejo de la Unión Europea, hay que indicar que el 28 de enero la Presidencia del Consejo activaba el dispositivo RPIC en modo de puesta en común de información ${ }^{34}$.

Quince días después tiene lugar un Consejo de Sanidad extraordinario que adopta una serie de Conclusiones sobre la COVID-19 ${ }^{35}$. Entre sus aspectos más relevantes destaca el reconocimiento de que "brotes de nuevas enfermedades transmisibles, como la COVID-19, constituyen amenazas para la salud pública que pueden llegar a tener alcance mundial, en particular debido a los volúmenes elevados y a la frecuencia de los viajes internacionales en un mundo globalizado." Asimismo, toma nota del "Plan Estratégico de Preparación y Respuesta relativo al 2019-nCoV", que aspira a detener la propagación ${ }^{36}$ del COVID-19 en China y otros países, a la vez que acentúa la importancia de las acciones rápidas emprendidas conjuntamente con la Comisión, en el marco del Reglamento Sanitario Internacional (RSI 2005) de la OMS, a través de la coordinación de las medidas de respuesta - como la búsqueda de contactos y la comunicación de riesgos-, y del intercambio de información sobre medidas nacionales de prevención y preparación en el marco del Comité de Seguridad Sanitaria y del SAPR, a fin de proteger la salud pública. Igualmente, tiene en cuenta la "Resolución Fortalecimiento de la preparación frente a emergencias sanitarias en aplicación del RSI 2005", aprobada por el Consejo Ejecutivo de la OMS el 8 de febrero de 2020.

A esta reunión siguen otras de carácter extraordinario con el objetivo de garantizar una respuesta coordinada. A tal fin, los ministros acuerdan centrarse en una serie de aspectos vinculados con el aumento de la sensibilización sobre la COVID-19; el refuerzo de la protección de las personas en situación de riesgo; el establecimiento de medidas de contención, en particular las relativas

\footnotetext{
${ }^{33}$ Faramiñan Gilbert: op.cit., p. 17. Del mismo autor, "Europa ante el desafío global de la pandemia COVID-19, en Boletín de la Academia de Yuste, 2020, n. 4, pp. 1-17.

${ }^{34} \mathrm{https}: / / \mathrm{eu} 2020 . \mathrm{hr} /$ Home/OneNews?id=160.

35 https://data.consilium.europa.eu/doc/document/ST-6038-2020-INIT/es/pdf

36 Publicado por la OMS el 3 de febrero de 2020, disponible en https://www.who.int/docs/defaultsource/coronaviruse/srp-04022020.pdf
}

Araucaria. Revista Iberoamericana de Filosofí, Política, Humanidades y Relaciones Internacionales, año $22, \mathrm{n}^{\circ} 45$. Tercer cuatrimestre de 2020. Pp. 337-357. ISSN 1575-6823 e-ISSN 2340-2199 https://dx.doi.org/10.12795/araucaria.2020.i45.14 
al asesoramiento basado en datos contrastados sobre los viajes hacia y desde las zonas de riesgo ${ }^{37}$; y la disponibilidad de los medicamentos en toda la UE y el desarrollo de una estrategia farmacéutica para Europa ${ }^{38}$, acorde con la Resolución 74/274 de la Asamblea General de las Naciones Unidas titulada "Cooperación Internacional para garantizar el acceso mundial a los medicamentos, vacunas y el equipo médico con los que hacer frente a la COVID-19"39.

Con motivo de la Propuesta de la Comisión Europea relativa a un Reglamento por el que se crea el "Cuarto programa de salud ("La UE por la Salud") para el período 2021-2027”, se vuelven a reunir los Ministros de Sanidad ${ }^{40}$. Aparte de acoger esta Propuesta, los ministros consideran esencial que Europa otorgue una mayor prioridad a la salud. La protección de sus ciudadanos exige conformar unos sistemas sanitarios más sólidos y resistentes en toda la UE, y lograr una mayor disponibilidad de medicamentos, de material médico y de protección y de equipos médicos de urgencia, así como un mayor intercambio de datos y una colaboración más estrecha entre los Estados miembros.

Por su parte, la actuación de la Comisión Europea no se hizo esperar. Así, desde el pasado 9 de enero viene trabajando para contener la propagación del coronavirus, proteger y salvar vidas, apoyar los sistemas sanitarios nacionales, y contrarrestar las repercusiones socioeconómicas de la pandemia ${ }^{41}$, a través de una serie de medidas distribuidas en cinco grandes bloques: a) medidas económicas; b) apoyo a la investigación para el tratamiento, el diagnóstico y las vacunas; c) salud pública; d) fronteras y movilidad; e) lucha contra la desinformación.

\footnotetext{
${ }^{37}$ https://eu2020.hr/Home/OneNews?id=201

38 Videoconferencia de los Ministros de Sanidad, 12.5.2020, disponible en https://eu2020.hr/ Home/OneNews?id=281

${ }^{39}$ Resolución aprobada por la Asamblea General el 20 de abril de 2020. En ella, el órgano principal subraya como "la disponibilidad, accesibilidad, aceptabilidad y asequibilidad de los productos sanitarios de calidad garantizada son fundamentales para hacer frente a la pandemia". Además, alienta a los Estados Miembros a que colaboren para aumentar la financiación de la investigación y el desarrollo de vacunas y medicamentos, y reforzar la coordinación con miras a un rápido desarrollo, fabricación y distribución de diagnósticos, medicamentos antivirales, equipo de protección personal y vacunas, respetando los objetivos de eficacia, seguridad, equidad, accesibilidad y asequibilidad. Por otra parte, reconoce "la importancia de la cooperación internacional y el multilateralismo eficaz para ayudar a garantizar que todos los Estados dispongan de medidas nacionales eficaces de protección, acceso a los suministros médicos, medicamentos y vacunas vitales, y un flujo suficiente de ellos, a fin de reducir al mínimo los efectos negativos en todos los Estados afectados y evitar los rebrotes de la pandemia".

${ }^{40}$ Videoconferencia de los Ministros de Sanidad, 12.6.2020, disponible en https://www.consilium. europa.eu/es/meetings/epsco/2020/06/12/

${ }^{41}$ El 9 de enero la Dirección General de Salud y Seguridad Alimentaria abre una notificación de alerta en el Sistema de Alerta Precoz y Respuesta (SAPR). Desde entonces la mayoría de los Estados miembros comparten información sobre las medidas de respuesta y comunicación. La cronología de las actuaciones de la Comisión esta disponible en https://ec.europa.eu/info/live-work-travel-eu/health/ coronavirus-response/timeline-eu-action es
} 
De manera particular nos centraremos en dos de estas medidas, comenzando por las que afectan al apoyo a la investigación para el tratamiento, el diagnóstico y las vacunas. En esta línea, la Comisión ha movilizado 546,53 millones de euros para desarrollar vacunas, nuevos tratamientos, pruebas de diagnóstico y sistemas médicos dirigidos a evitar la propagación del coronavirus y salvar vidas ${ }^{42}$.

A ello se suma su Estrategia en materia de vacunas para acelerar el desarrollo y la disponibilidad de vacunas en un plazo de entre 12 y 18 meses $^{43}$. La Estrategia propone un planteamiento común de la UE, asentado en dos pilares fundamentales dirigidos, por un lado, a garantizar la producción de vacunas en la UE y su suministro a los Estados miembros en cantidad suficiente mediante compromisos anticipados de mercado con los productores de vacunas; y, por el otro, a adaptar el marco reglamentario de la UE a la situación de urgencia actual, aprovechando la flexibilidad de la legislación europea para acelerar el desarrollo, la autorización y la disponibilidad de las vacunas. Sus objetivos centrales son: i) garantizar la calidad, la seguridad y la eficacia de las vacunas; ii) el acceso rápido a las vacunas para los Estados miembros y su población, liderando al mismo tiempo el esfuerzo de solidaridad mundial; iii) el acceso equitativo a una vacuna asequible lo antes posible.

La Estrategia también prevé la celebración de acuerdos individuales por parte de la Comisión con productores de vacunas en nombre de los Estados miembros ${ }^{44}$. Además, la Comisión financiará una parte de los costes iniciales afrontados por dichos productores, a través de compromisos anticipados de mercado a cambio del derecho a comprar un número determinado de dosis de vacunas durante un período dado ${ }^{45}$.

Respecto al bloque de la salud pública, la Comisión creó la Reserva estratégica común de equipos médicos de emergencia (rescEU), que permite a la Institución comunitaria adquirir material directamente en nombre de

${ }^{42}$ Esto incluye: i) 41 nuevos proyectos de investigación en los que participan 498 equipos de investigadores de toda Europa y el resto del mundo; ii) contribuciones destinadas, entre otros, a la Coalición para la Innovación en la Preparación frente a Epidemias, al Programa de cooperación de los países europeos y de los países en desarrollo sobre ensayos clínicos, o a la Iniciativa sobre Medicamentos Innovadores; iii) contribuciones para las ideas pioneras relacionadas con el coronavirus.

${ }^{43}$ Disponible en https://ec.europa.eu/commission/presscorner/detail/en/ip $20 \quad 1103$

${ }^{44}$ La Comisión alcanzó un primer acuerdo con la empresa farmacéutica AstraZ̄eneca para comprar una posible vacuna contra la COVID-19, así como para donarla a países de renta media y baja o redistribuirla a otros países europeos. Esta vacuna experimental ya se encuentra en la fase II/III de ensayos clínicos a gran escala, después de obtener resultados prometedores en la fase I/II con respecto a la seguridad y la inducción de la respuesta inmune. Información disponible en https:/ec.europa. $\mathrm{eu} /$ commission/presscorner/detail/\%5Beuropa_tokens:europa_interface_language $\% 5 \mathrm{D} / \mathrm{ip} \_20 \_1524$

${ }^{45}$ La financiación será a cargo en gran medida del "Instrumento para la Prestación de Asistencia Urgente". Además, brindará apoyo adicional mediante préstamos del Banco Europeo de Inversiones. Ver https:/ec.europa. eu/info/live-work-travel-eu/health/coronavirus-response/emergency-support-instrument es\#general 
los Estados miembros ${ }^{46}$, y financiar y coordinar el transporte de equipos médicos y de pacientes en regiones transfronterizas. La tarea de distribución de los equipos se encomienda al Centro de Coordinación de la Respuesta a Emergencias.

Otra de las medidas tomadas por la Comisión en materia de salud pública ha sido la creación de un equipo de expertos científicos integrado por epidemiólogos y virólogos independientes con un doble mandato, dirigido, por un lado, a formular directrices sobre medidas de gestión de riesgos coordinadas y con base científica; y, por el otro, a realizar tareas de asesoramiento sobre diversas medidas.

A estas medidas se suman otras, entre las que destacan: i) las Directrices de la Comisión para los Estados miembros relativas al suministro, la distribución y el uso racional de los medicamentos vitales para el tratamiento de los pacientes de coronavirus y de los medicamentos cuya disponibilidad pueda estar en riesgo: ii) las Orientaciones sobre las metodologías para las pruebas de coronavirus; iii) la autorización a escala de la UE de comercialización condicional del Remdesivir, como primer medicamento para el tratamiento del coronavirus.

\section{Reflexión final}

Para concluir y a modo de reflexión final, quisiera destacar en primer lugar que, pese a sus luces y sombras, tanto la Unión Europea como sus Estados miembros a través de todos los instrumentos a su alcance en el marco de sus respectivas competencias, han priorizado la salud de la población. La salud continúa siendo competencia exclusiva de los Estados miembros, de manera que la capacidad de la UE para hacer frente a la expansión de la pandemia, como examinamos, se limita a una combinación de mecanismos dirigidos a la adopción de medidas adecuadas para su contención.

Los Estados miembros durante el avance y propagación de la pandemia han intentado coordinarse para lograr mantener de la mejor forma posible la situación epidemiológica en toda la Unión. Las medidas de salud pública adoptadas por sus miembros han ayudado a reducir el número de nuevas infecciones hasta un nivel gestionable por los sistemas sanitarios, lo que a su vez ha permitido el levantamiento progresivo de algunas de las restricciones impuestas y la reapertura de la mayoría de las actividades, guiados por la "Hoja de ruta común europea para el levantamiento de medidas de contención de la COVID-19"47.

${ }^{46}$ https://ec.europa.eu/echo/files/aid/countries/factsheets/thematic/2020 rescEU MFF en.pdf

${ }_{47}$ Disponible en https://ec.europa.eu/info/sites/info/files/joint_eu_roadmap_lifting_covid19 
Con todo la pandemia de COVID-19 ha provocado una crisis sin precedentes tanto en la Unión Europea como en el resto del mundo. Como nos recuerda la Comisaria de Salud y Seguridad Alimentaria: "Hemos recorrido un largo camino desde que la pandemia de COVID-19 alcanzara su punto álgido, pero el virus sigue circulando. La vigilancia, la preparación y la coordinación son indispensables para prevenir los brotes generalizados. Hoy hacemos un llamamiento en favor de una acción firme y común para proteger a nuestros ciudadanos y, para lograrlo, brindaremos nuestro apoyo a los Estados miembros. Es nuestra responsabilidad garantizar que estamos totalmente preparados. Ahora no es el momento de bajar la guardia"48.

Y en esta línea recalca el Presidente del Parlamento Europeo "La pandemia nos ha dado responsabilidades y deberes nuevos: la responsabilidad de tomar decisiones y el deber de hacerlo teniendo en cuenta el interés de la mayoría y no de unos pocos. Es evidente dónde debemos invertir: en la economía verde, sanidad, educación y en derechos digitales, democráticos y sociales" $"$.

Trasciende por tanto la necesidad de que la UE y sus Estados miembros continúen trabajando y aunando esfuerzos frente a los rebrotes del COVID-19 que, en estos momentos, continúan apareciendo en todo el territorio de la Unión, o de otras epidemias o pandemias que puedan aparecer en el futuro. Para ello será necesario, "extraer todas las enseñanzas de la actual crisis y empezar a reflexionar sobre la resiliencia que tienen nuestras sociedades cuando afrontan tales acontecimientos... [H]a llegado el momento de instaurar en la UE un sistema de gestión de crisis más ambicioso y de mayor alcance" 50 . Corresponde a la Comisión en estrecha coordinación con los Estados miembros y las agencias de la Unión presentar propuestas al respecto. En este sentido, la Institución europea ha establecido una serie de medidas inmediatas para reforzar la preparación sanitaria de la UE frente a los rebrotes de COVID-19 y evitar tener que volver a imponer restricciones a gran escala como el confinamiento ${ }^{51}$.

Compartimos con Roldán Barbero que "La Europa que protege ha de tener, además, una dimensión exterior, inextricablemente unida a la dimensión interior, como la regulación actual de las fronteras pone de manifiesto, en la

\footnotetext{
containment measures es.pdf

${ }^{48} \mathrm{En}$ https://ec.europa.eu/commission/presscorner/detail/es/ip $20 \quad 1340$

49 Disponible en https://www.europarl.europa.eu/the-president/en/newsroom/time-to-decide-theconditions-of-the-european-parliament

${ }^{50}$ Declaración conjunta de los miembros del Consejo Europeo de 26.3.2020, disponible en https:// www.consilium.europa.eu/media/43098/26-vc-euco-statement-es.pdf

51 Comunicación de la Comisión al Parlamento Europeo, al Consejo, al Comité Económico y Social Europeo y al Comité de las Regiones: Preparación sanitaria de la Unión a corto plazo frente a brotes de COVID-19, COM (2020) 318 final, 15.7.2020, disponible en https://eur-lex.europa.eu/ resource.html?uri=cellar:f6fbab84-c749-11ea-adf7-01aa75ed71a1.0010.02/DOC 1\&format=PDF
} 
cual aparece, con la pandemia, una nueva acepción del concepto "país seguro" ... La "soberanía" europea ha de manifestarse en ambos planos, sosteniendo un orden jurídico multilateral liberal, ejerciendo de verdadera potencia normativa...Europa debe articular "una política exterior sólida y firme"

Por otro lado, conviene subrayar que esta pandemia ha puesto de relieve la "dimensión "internacional" de los problemas relacionados con la salud, que no pueden abordarse desde una perspectiva estrictamente nacional y que precisan, necesariamente, de la cooperación multilateral'. La salud global exige ser considerada como un bien público global" ${ }^{53}$. Debemos propiciar "la construcción de una consciencia pública internacional solidaria de todos y especialmente de los actores de mayor peso"54. Y en esta línea, la Comisión Europea debe continuar coordinándose con otros actores mundiales, como la ONU y la OMS, a fin de garantizar la respuesta internacional que requiere la amenaza de la COVID-19 u otras futuras pandemias. Como subraya la propia Comisión, esto debe acompañarse "de esfuerzos por abordar los efectos socioeconómicos de esta crisis, que amenazan con tirar por tierra los avances logrados en la consecución de los Objetivos de Desarrollo Sostenible, así como de inversión en la resiliencia de los países socios, en particular a través del refuerzo de los sistemas sanitarios, apuntalando así la recuperación mundial. Esta es la única forma de poner fin a la actual crisis pandémica en la Unión y en el resto del mundo" 55 .

La crisis del COVID-19 muestra los desafíos a los que se enfrenta Europa. El futuro de Europa exige estar preparados para futuras crisis sanitarias. Con este objetivo para responder a la crisis actual y a las que puedan venir, en el ámbito de la salud, ya se propugna un nuevo enfoque europeo basado en la "soberanía sanitaria estratégica", que posibilite alcanzar una industria de salud europea estratégicamente posicionada que, respetando plenamente la responsabilidad de los Estados miembros por sus sistemas de seguridad y salud, actualice la dimensión europea de la atención médica y reduzca la dependencia que sufre Europa $^{56}$.

52 Javier Roldán Barbero, "Reflexiones de un internacionalista sobre la pandemia. /5. ¡EUROPA, EUROPA!", Aquiescencia. Blog de Derecho Internacional, disponible en https://aquiescencia. net/2020/06/30/reflexiones-de-un-internacionalista-sobre-la-pandemia-5-europa-europa-por-javierroldan-barbero/junio 30, 2020

53 Xabier Pons Rafols, "La COVID-19, la salud global y el Derecho internacional: Una primera aproximación de carácter institucional”, REEI, No 39, 2020, p. 4, disponible en http://www.reei.org

54 Alberto César Moreira, "Soberanía estatal y cooperación internacional. Reflejos del Derecho internacional frente al desafío de la COVID-19”, Cuadernos de Derecho Público, Volumen 8, 2020, p. 88.

55 Comunicación de la Comisión al Parlamento Europeo, al Consejo, al Comité Económico y Social Europeo y al Comité de las Regiones, Preparación sanitaria de la Unión a corto plazo..., cit., pp. $15-16$.

${ }^{56}$ Recogida en la "Iniciativa franco-germana para la recuperación europea de la crisis coronavirus", adoptada el 18 de mayo de 2020, disponible en https://www.bundeskanzlerin.de/resource/blob/6567 34/1753772/414a4b5a1ca91d4f7146eeb2b39ee72b/2020-05-18-deutsch-franzoesischer-erklaerungeng-data.pdf 
Hoy más que nunca se reconoce que el proyecto europeo nacido hace más de sesenta años debe continuar. "A quien gobierna le corresponde discernir los caminos de la esperanza - este es su cometido: discernir los caminos de la esperanza-, identificar los procesos concretos para hacer que los pasos realizados hasta ahora no se dispersen, sino que aseguren un camino largo y fecundo... Es necesario volver a pensar en modo europeo, para conjurar el peligro de una gris uniformidad o, lo que es lo mismo, el triunfo de los particularismos" $" 57$.

${ }^{57}$ Discurso del Santo Padre Francisco a los Jefes de Estado y de Gobierno de la Unión Europea presentes en Italia para la celebración del 60 Aniversario del Tratado de Roma, 24 de marzo de 2017, disponible en http://www.vatican.va/content/francesco/es/speeches/2017/march/documents/papafrancesco_20170324_capi-unione-europea.html\#_ftn1 


\section{Referencias bibliográficas:}

Benedicto, Miguel Ángel, Los retos de Europa y su futuro tras la pandemia de la COVID-19. Documento de Opinión IEEE 72/2020, 14 págs. Disponible en http://www.ieee.es/Galerias/fichero/docs_opinion/2020/ DIEEEO72 2020MIGBEN retosEU.pdf

Cabrera-Gaytán, David Alejandro, Vargas-Valerio, Alfredo y Grajales-Muñiz, Concepción, "Infección del nuevo coronavirus: nuevos retos, nuevos legados", en Revista Médica del Instituto Mexicano del Seguro Social, 2014, 452(4), pp. 438-441.

Calvete Oliva, Antonio, "Estrategia de Salud de la Unión Europea: Salud pública para las personas Europas”, Revista Española de Salud Pública, $\mathrm{N}^{\mathrm{o}}$ 3, mayo-junio 2008, pp. 273-281.

Campins Eritja, Mar, "La Unión Europea y la protección de la salud pública", en Xavier Pons Rafolds, ed.: Salud Pública y Derecho Internacional, Madrid, Marcial Pons, 2010, pp. 375-414.

Catalán Matamoros, Daniel J., "Mecanismos de prevención y control de las enfermedades infecciosas en la Unión Europea: El Centro Europeo para la Prevención y el Control de enfermedades”, en Xavier Pons Rafols, ed.: Salud Pública y Derecho Internacional, Madrid, Marcial Pons, 2010, pp. 415-424.

Cique Moya, Alberto, "Capacidades sanitarias militares en la prevención y lucha frente a emergencias epidémicas", en Cuadernos de Estrategia 203, Emergencias pandémicas en un mundo globalizado: Amenazas a la seguridad, Madrid, Instituto Español de Estudios Estratégicos, 2020, pp. 207-253, disponible en http:/www.ieee.es/publicaciones-new/cuadernosde-estrategia/2020/Cuaderno 203.html

Consejo General del Colegio de Farmacéuticos, COVID-19. Informe Técnico, marzo, 2020.

De la Flor, José Luis, "La seguridad sanitaria global a debate. Lecciones críticas aprendidas de la 24. 'EVE", en Cuestiones de seguridad internacional en el África del siglo XXI Comillas Journal of International Relations, Núm. 13 (2018), pp. 49-62.

Faramiñan Gilbert, Juan Manuel, "La protección de la salud pública y el respeto de las libertades individuales ante el COVID-19", Freedom, Security \& Justice: European Legal Studies. Rivista quadrimestrale on line sullo Spazio europeo di libertà, sicurezza e giustizia, 2020, n. 2, pp. 1-21, disponible en http://www.fsjeurostudies.eu

"Europa ante el desafío global de la pandemia COVID-19, en Boletín de la Academia de Yuste, 2020, n. 4, pp. 1-17. 
Fuente Cobo, Ignacio, El mundo después de la pandemia: el nuevo orden no será chino. Documento de Opinión IEEE 33/2020, 11 págs. Disponible en http://www.ieee.es/Galerias/fichero/docs_opinion/2020/ DIEEEO33 2020IGNFUE mundo.pdf;

Martínez Hernández, Juan, "Pandemias y bioamenazas globales del siglo XXI”, ARI no 42/2016, Real Instituto Elcano, 30/VI/2016, 7 págs. Disponible en http:/www.realinstitutoelcano.org/wps/portal/rielcano es/ contenido?WCM GLOBAL_CONTEXT=/elcano/elcano es/zonas es/ ari42-2016-martinezhernandez-pandemias-bioamenazas-globalessiglo-21

Moreira, Alberto César, "Soberanía estatal y cooperación internacional. Reflejos del Derecho internacional frente al desafío de la COVID-19”, Cuadernos de Derecho Público, Volumen 8, 2020, pp. 71-91.

Pérez Bernárdez, María del Carmen, Las relaciones de la Unión Europea con organizaciones internacionales: Análisis jurídico de la práctica constitucional, Madrid, Dirección General de Universidades, Consejería de Educación, 2003.

Pons Rafols, Xabier, "La COVID-19, la salud global y el Derecho internacional: Una primera aproximación de carácter institucional”, REEI, $\mathrm{N}^{\circ} 39,2020$, 29 págs. Disponible en http://www.reei.org

Roldán Barbero, Javier, "Reflexiones de un internacionalista sobre la pandemia. 15. ¡EUROPA, EUROPA!”, Aquiescencia. Blog de Derecho Internacional, disponible en https://aquiescencia.net/2020/06/30/reflexiones-de-uninternacionalista-sobre-la-pandemia-5-europa-europa-por-javier-roldanbarbero/junio 30, 2020.

Sobrino Heredia, José Manuel, "La política de salud pública en la Unión Europea", Anuario da Facultade de Dereito da Universidade da Coruña, 1998, pp. 547-581. 
